\title{
Higgs boson measurements in final states with taus at CMS
}

\author{
Daniel Winterbottom,* and on behalf of the CMS Collaboration \\ Imperial College London \\ E-mail: daniel.winterbottom@cern.ch
}

The latest Higgs boson measurements in final states with tau leptons are reviewed. These measurements are based on $137 \mathrm{fb}^{-1}$ of data collected with the CMS experiment at centre-of-mass energy of $13 \mathrm{TeV}$. An overall signal strength of $0.85_{-0.11}^{+0.12}$ with respect to the standard model prediction is observed. A measurement of the CP properties of the tau Yukawa coupling is also presented for the first time. The mixing angle between CP-even and CP-odd tau Yukawa couplings was found to be $4 \pm 17^{\circ}$. The observed significance of the separation between the CP-even and CP-odd hypotheses is 3.2 standard deviations.

40th International Conference on High Energy physics - ICHEP2020

July 28 - August 6, 2020

Prague, Czech Republic (virtual meeting)

\footnotetext{
${ }^{*}$ Speaker
} 


\section{Introduction}

Measurements of Higgs boson decays to tau leptons offer a direct probe of the properties of the Yukawa coupling to tau leptons. The CP properties of the $\tau$ Yukawa coupling can also be probed by measuring angular correlations between tau lepton decay products. The $\mathrm{H} \rightarrow \tau \tau$ final state is also sensitive to other Higgs couplings via its production modes such as gluon fusion $(\mathrm{ggH})$ and vector boson fusion (VBF). The relatively high branching fraction for Higgs decay to tau leptons coupled with the reasonably good signal to background ratios makes this final state particularly useful for measuring sub-dominant production processes such as VBF, and gives good sensitivity to less populated regions of phase space where the Higgs is produced with a large transverse momentum and/or is accompanied by multiple jets. Such measurements can thus complement differential measurements performed in other final states, such as in the simplified template cross section (STXS) scheme [1]. In these proceedings the latest $\mathrm{H} \rightarrow \tau \tau$ measurements conducted by the CMS experiment [2] using $137 \mathrm{fb}^{-1}$ of data at a centre-of-mass energy of $13 \mathrm{TeV}$ are reviewed.

\section{Cross section measurements}

Tau leptons are unstable and decay close to the primary interaction point inside the beam-pipe either hadronically or leptonically, accompanied by one or two neutrinos respectively. The leptons are selected by requiring candidates to pass a set of identification and isolation requirements to reduce the contribution of misidentified leptons. Similarly, hadronic taus are required to pass a multivariate discriminant designed to reject misidentified taus arising from jets. The neutrinos are undetectable but the system can be partially reconstructed by the missing transverse momentum in the event, $\vec{p}_{\mathrm{T}}^{\mathrm{miss}}$. Events are subdivided into four channels depending on how the pair of taus decay: $\tau_{h} \tau_{h}, \tau_{\mu} \tau_{h}, \tau_{e} \tau_{h}, \tau_{e} \tau_{\mu}$, where $\tau_{\mu}$ and $\tau_{e}$ are shorthand for muonic- and electronic-ally decaying taus and $\tau_{h}$ refers collectively to all hadronic decays. All ditau pairs are additionally required to have opposite-sign electric charges.

In order to maximise the sensitivity to the Higgs signal and target different production modes, events are categorised into three categories based on the jet multiplicity. These categories are then further subdivided to match the STXS bin boundaries to enhance the sensitivity to the STXS measurements. In each category two dimensional discriminants are used to extract the Higgs signal. One observable is always the ditau mass, $m_{\tau \tau}$, which is estimated approximately combining information from visible decay products with $\vec{p}_{\mathrm{T}}^{\text {miss }}$ [3]. The second observable is chosen on a per-category basis to enhance the separation between signal and background and to align further with STXS bins. Full details on the analysis strategy can be found in Ref. [4].

The main background to the analysis are from $\mathrm{Z} / \gamma^{*} \rightarrow \tau \tau$ events which is estimated using the embedding method [5]. The second largest background is due to misidentified $\tau_{h}$ candidates arising from jets, which is estimated from a sideband region in which the $\tau_{h}$ candidates fail the nominal identification requirements [6]. The contribution of QCD multi-jet events in the $\tau_{e} \tau_{\mu}$ is estimated from a data-driven method exploiting same-sign $\tau_{e} \tau_{\mu}$ pairs. All remaining smaller background processes as well as the Higgs signal are estimated using simulation.

The results are extracted by means of a binned maximum likelihood fit combining all channels and categories. Systematic uncertainties of experimental and theoretical origin are included in the 

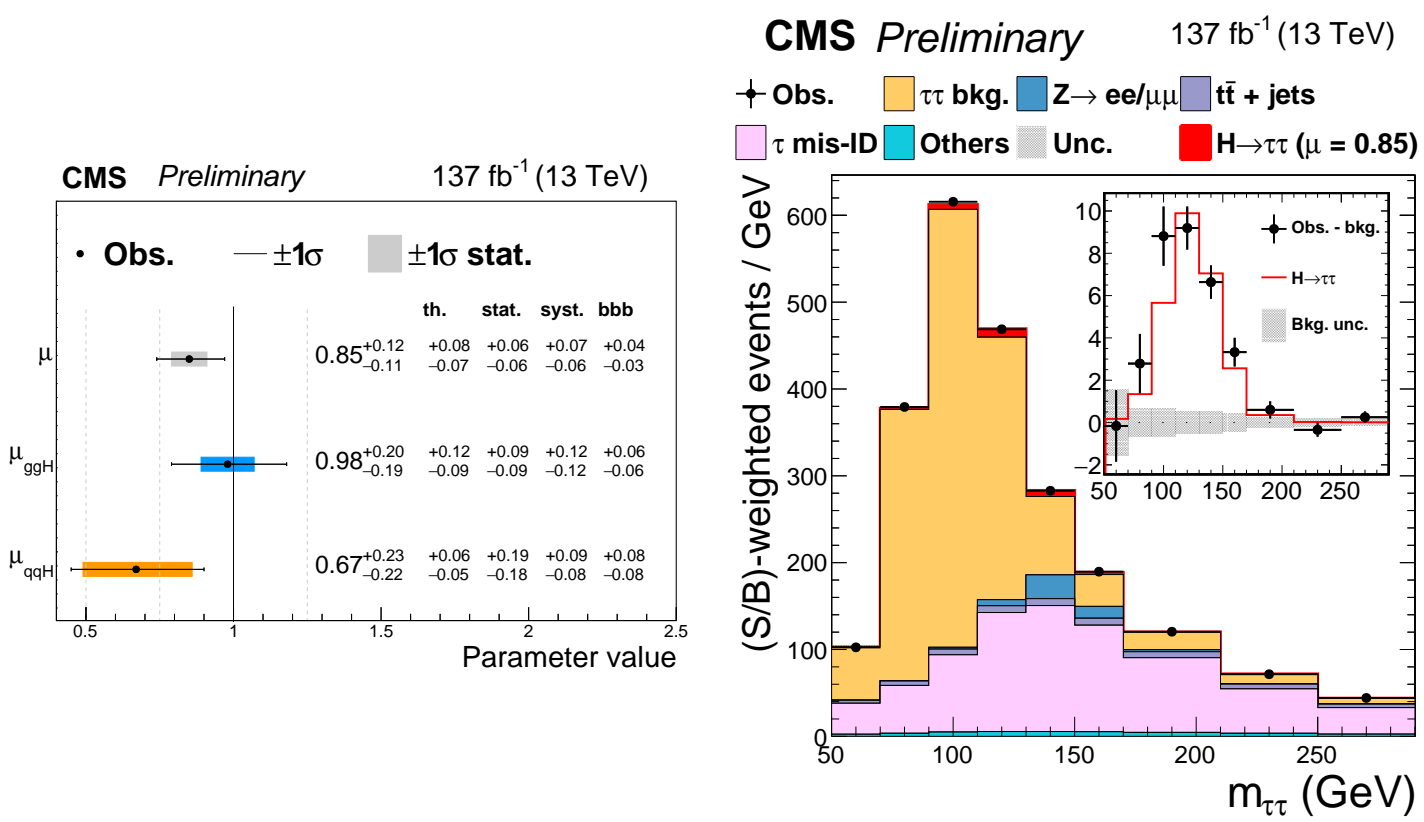

Figure 1: Left: Observed results of the fit to the production mode signal strength modifiers. Right: Observed and expected $m_{\tau \tau}$ distribution obtained by weighting every $m_{\tau \tau}$ distribution of each category, year, and final state by the ratio between the signal and background yields. The inset shows the difference between the observed data and the expected background distributions, together with the signal expectation. Figures taken from Ref. [4].

signal and background models. The inclusive Higgs cross section is measured by introducing a signal strength modifier $\mu$ that scales the cross section relative to the SM expectation. The best fit value of $\mu$ is measured to be $0.85_{-0.11}^{+0.12}$, consistent with the $\mathrm{SM}$ expectation $(\mu=1)$.

The cross sections for the ggH and VBF production processes are measured simultaneously by introducing two separate signal strength modifiers, $\mu_{\mathrm{ggH}}$ and $\mu_{\mathrm{qqH}}$, which are determined to be $\mu_{\mathrm{ggH}}=0.98{ }_{-0.19}^{+0.20}$ and $\mu_{\mathrm{qqH}}=0.67_{-0.22}^{+0.23}$. A summary of the inclusive and by-process cross section measurements is shown in Fig. 1. The observed and expected $m_{\tau \tau}$ distribution, where all categories and final states are have been weighted by the ratio of the signal to background yields and combined into a single distribution, is also shown in Fig. 1. The best-fit Higgs signal is shown stacked on top of the background distribution in red and can be visualised more clearly in the top right inset of the figure where the expected background contribution has been subtracted from the data. A clear peak is observed close to a mass of $125 \mathrm{GeV}$. Measurements of the stage 0 and stage 1 STXS parameters are also presented in Fig. 2. Some STXS bins have been merged following the so-called process based merging scheme from Ref. [4].

\section{CP measurement}

The CP properties of the $\tau$ Yukawa coupling have also been measured for the first time. This analysis targets the two most sensitive channels, $\tau_{h} \tau_{h}$ and $\tau_{\mu} \tau_{h}$. These channels are further subdivided depending on the specific $\tau_{h}$ decay modes, including direct decays to a single charged 


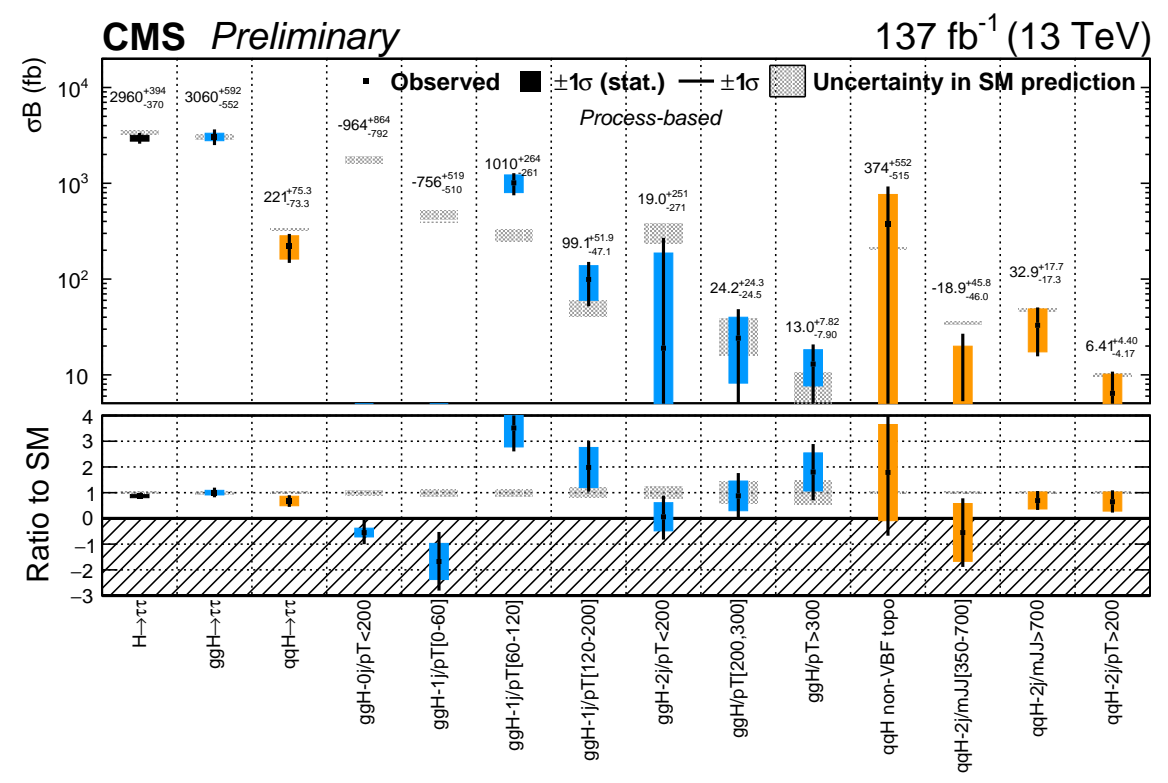

Figure 2: Products of the cross section and branching fraction measured for the inclusive, stage- 0 , and stage-1 STXS parameters. The process-based stage-1 merging described in Ref. [4] is employed. The ggH processes are indicated in blue while the $\mathrm{qqH}$ are indicated in yellow. Figure taken from Ref. [4].

pion as well as the decays to charged and neutral pions via intermediate $\rho$ resonances. The specific definitions of the $\mathrm{CP}$ sensitive variables depend on the particles in the final state and for convenience will be collectively referred to as $\phi_{\mathrm{CP}}$. For $\tau_{h} \tau_{h}$ events where both $\tau_{h}$ decays involve intermediate $\rho$ resonances angular correlations between the visible decay products are sensitive to the CP properties. For example in the final state where two $\tau_{h}$ candidates decay to a charged and neutral pion via a $\rho$ resonance $\left(\tau^{+} \tau^{-} \rightarrow \rho v \rho v \rightarrow \pi^{+} \pi^{0} v \pi^{-} \pi^{0} v\right) \phi_{\mathrm{CP}}$ is defined as the angle between the two $\pi^{0}$ 's in the rest frame of the $\pi^{+} \pi^{-}$system. For $\tau_{\mu}$ and $\tau_{h} \rightarrow \pi^{ \pm} v$ events with only one visible decay product the tau impact parameter vector, defined as the vector that points from the primary interaction vertex (PV) to the point of closest approach of the $\pi^{ \pm} / \mu$ track to the $\mathrm{PV}$, is used in place of the $\pi^{0}$ momentum vector. More information about the $\phi_{\text {CP }}$ definitions can be found in Ref [7].

Multivariate methods based on neural networks (NN) and boosted decision trees (BDT) are used to provide separation of the Higgs signal from the background. Two-dimensional discriminants are then used to extract the results, where one observable is $\phi_{\mathrm{CP}}$ and the other is the NN/BDT output score. The methods used to estimate the signal and background processes are similar to those described in Section 2.

The CP properties of the Yukawa interaction are parametrised in terms of an effective mixing angle $\phi_{\tau \tau}$, defined as $\phi_{\tau \tau}=\arctan \left(\frac{\tilde{\kappa}_{\tau}}{\kappa_{\tau}}\right)$, where $\tilde{\kappa}_{\tau}$ and $\kappa_{\tau}$ are the reduced (relative to the SM tau Yukawa coupling) CP-odd and CP-even $\tau$ Yukawa couplings respectively. A measured value of $\phi_{\tau \tau}=0^{\circ}$ would imply a CP-even Higgs boson as predicted by the SM, $\phi_{\tau \tau}=90^{\circ}$ corresponds to a CP-odd boson, and $0^{\circ}<\left|\phi_{\tau \tau}\right|<90^{\circ}$ corresponds to a $\mathrm{CP}$ violating admixture. The value of $\phi_{\tau \tau}$ is extracted by a binned maximum likelihood fit, the negative-log likelihood scan is shown in 

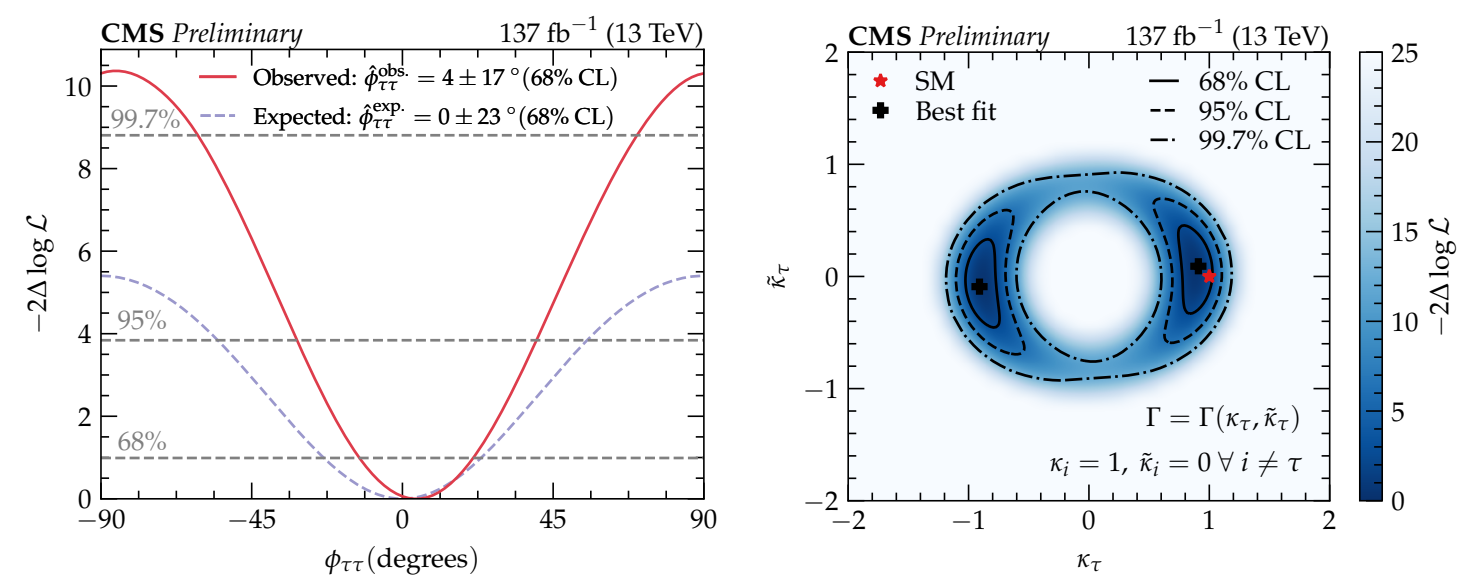

Figure 3: Left: The negative log-likelihood scan of $\phi_{\tau \tau}$. Right: Two-dimensional scan of the (reduced) CP-even $(\kappa)$ and CP-odd $(\tilde{\kappa}) \tau$ Yukawa couplings. Figures taken from Ref. [7].

Fig. 3. From this scan we extract an observed (expected) value for $\phi_{\tau \tau}$ of $4 \pm 17^{\circ}\left(0 \pm 23^{\circ}\right)$. A two-dimension scan of $\tilde{\kappa}_{\tau}$ and $\kappa_{\tau}$ is also shown in Fig. 3. In Fig. 4 we display the data of the three most sensitive channels together with CP-even and CP-odd predictions. The data has been weighed for an enhanced visual interpretation. The data clearly favour the CP-even scenario. The CP-even scenario is preferred to the CP-odd at the level of 3.2 (2.3) standard deviations.

\section{Summary}

The latest $\mathrm{H} \rightarrow \tau \tau$ measurements conducted by the CMS experiment have been reviewed. The signal strength modifiers for inclusive Higgs production as well as for the ggH and VBF production modes have been measured to be $0.85_{-0.11}^{+0.12}, \mu_{\mathrm{ggH}}=0.98_{-0.19}^{+0.20}$, and $\mu_{\mathrm{qqH}}=0.67_{-0.22}^{+0.23}$ respectively. Differential measurements in the context of the STXS framework have also been presented. Measurements of the CP properties of the $\tau$ Yukawa coupling have also been performed for the first time. The effective mixing angle was measured as $\phi_{\tau \tau}=4 \pm 17^{\circ}$. The CP-even scenario is preferred to the CP-odd at the level of 3.2 standard deviations. 


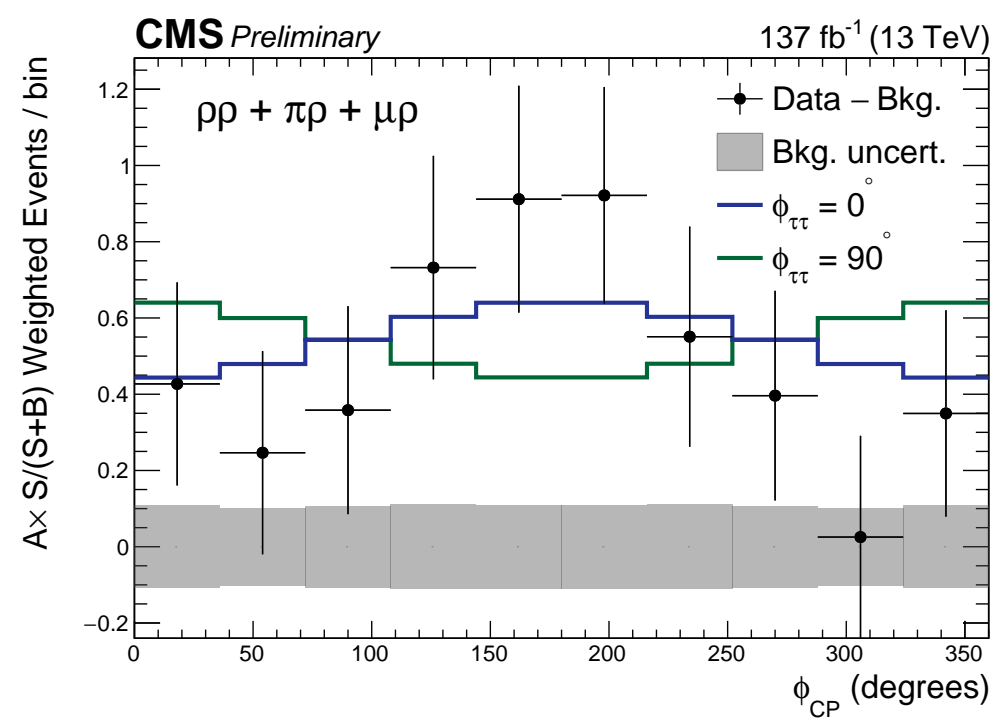

Figure 4: The $\phi_{\mathrm{CP}}$ distribution for the three most sensitive channels combined. The background is subtracted from the data. The events are weighed via $A S /(S+B)$, in which $S$ and $B$ are the signal and background rates, respectively, and $A$ is a measure for the average asymmetry between the scalar and pseudoscalar distributions. The scalar distribution is depicted in blue, while the pseudoscalar is displayed in green. In the predictions, the rate parameters are taken from their best-fit values. Figure taken from Ref. [7].

\section{References}

[1] D. de Florian et al., Handbook of LHC Higgs cross sections: 4. deciphering the nature of the Higgs sector, CERN Report CERN-2017-002-M

[2] CMS collaboration, S. Chatrchyan et al., The CMS experiment at the CERN LHC, JINST 3 S08004 (2008).

[3] L. Bianchini, J. Conway, E. K. Friis, and C. Veelken Reconstruction of the Higgs mass in $H \rightarrow \tau \tau$ Events by Dynamical Likelihood techniques, J. Phys. Conf. Ser. 513022035 (2014)

[4] CMS collaboration, Measurement of Higgs boson production in the decay channel with a pair of $\tau$ leptons, CMS-PAS-HIG-19-010

[5] CMS collaboration, A.M. Sirunyan et al., An embedding technique to determine $\tau \tau$ backgrounds in proton-proton collision data, JINST 14 P06032 (2019).

[6] CMS collaboration, A.M. Sirunyan et al., Measurement of the $\mathrm{Z} / \gamma^{*} \rightarrow \tau \tau$ cross section in $p p$ collisions at $\sqrt{s}=13 \mathrm{TeV}$ and validation of $\tau$ lepton analysis techniques, Eur. Phys. J. C 78, 708 (2018)

[7] CMS collaboration, Analysis of the CP structure of the Yukawa coupling between the Higgs boson and $\tau$ leptons in proton-proton collisions at $\sqrt{s}=13 \mathrm{TeV}$, CMS-PAS-HIG-20-006 